\title{
Nolana balsamiflua (Gaudich.) Mesa (Solanaceae), un arbusto vulnerable del desierto de Atacama dependiente de polinizadores para producir semillas
}

\section{Nolana balsamiflua (Gaudich.) Mesa (Solanaceae), a vulnerable shrub of the Atacama Desert dependent on pollinators for seed set}

\author{
Ana María Humaña, Alberto Jiménez \& Carlos E. Valdivia* \\ Laboratorio de Vida Silvestre, Departamento de Ciencias Biológicas y Biodiversidad, Universidad de Los Lagos, Casilla 933, \\ Osorno, Chile. \\ *carlos.valdivia@ulagos.cl
}

\begin{abstract}
Pollinator-dependency for reproduction is of great importance for plants. We evaluated the breeding system of Nolana balsamiflua, a threatened endemic shrub of the Atacama Desert, performing four pollination trials. No flower produced seeds by agamospermy, autogamy and geitonogamy. However, xenogamy accounted for $51.4 \%$ of fruiting, indicating its full dependency on exogenous pollen and pollinators for reproduction.
\end{abstract}

El sistema reproductivo de las plantas es un rasgo que condiciona los patrones reproductivos individuales y la viabilidad poblacional (Richards 1997). Consecuentemente, su determinación permite comprender aspectos ecológicos y evolutivos, así como establecer estrategias de conservación pertinentes en especies amenazadas por las actividades antropogénicas (Aizen \& Vázquez 2006).

En general, existen cuatro posibles vías para la producción de semillas (Richards 1997). La agamospermia es la vía reproductiva asexual mediante la cual las semillas son producidas en ausencia de polen. La autogamia es la reproducción mediante óvulos autofertilizados con polen producido por la misma flor, pero sin la mediación de polinizadores. La geitonogamia es la producción de semillas a través de la fertilización de los óvulos con polen producido por el mismo individuo, pero transportado por polinizadores. Finalmente, la xenogamia es la producción de semillas mediante la fecundación de los óvulos con polen proveniente de otros individuos de la población (Richards 1997).

La agamospermia y autogamia permiten a las plantas asegurar su reproducción, especialmente en ambientes con escasa diversidad y abundancia de polinizadores, como los desiertos (González \& Pérez 2010). Las plantas agamospérmicas y autógamas, además, son menos vulnerables a las perturbaciones de origen humano dada su independencia reproductiva (Aizen \& Vázquez 2006). El desierto de Atacama en Chile se caracteriza por presentar periodos de sequía de 2 a 5 años, seguidos de lluvias de corta duración y variables en cantidad (González \& Pérez
2010). Esto permite el crecimiento rápido de plantas e insectos polinizadores, los que pueden llegar a ser local y esporádicamente abundantes (González \& Pérez 2010). Allí, escasas investigaciones han evaluado los sistemas reproductivos de las plantas y su dependencia por polinizadores (pero véase González \& Pérez 2010), lo cual es desafortunado dado el fuerte estrés hídrico y las extendidas perturbaciones antropogénicas (e.g. minería) que enfrentan en este ambiente. En el Desierto de Atacama habitan numerosas especies endémicas, como Nolana divaricata (Lindl.) I.M. Johnst. y N. rupicola Gaudich., las que se reproducen parcialmente sin polinizadores (González \& Pérez 2010).

Nuestro objetivo es estimar el sistema reproductivo putativo del arbusto endémico y vulnerable Nolana balsamiflua (Gaudich.) Mesa en una población del desierto de Atacama en Chile. Dado que las plantas de ambientes estresantes usualmente producen semillas de manera autógama para asegurar su reproducción, esperamos que la autogamia, al igual que la xenogamia, sea potencialmente una importante vía reproductiva.

Desarrollamos el trabajo de campo entre diciembre de 2011 y marzo de 2012 en la "Quebrada del Diablo, Michilla" en la Región de Antofagasta, Chile $\left(22^{\circ} 42^{\prime} \mathrm{S}, 70^{\circ} 15^{\prime} \mathrm{W}\right.$, Fig. 1A). La vegetación corresponde al Matorral Desértico Tropical Costero de Ephedra americana Humb. \& Bonpl. ex Willd. y Eulychnia iquiquensis (K. Schum.) Britton \& Rose, que presenta extensas áreas desprovistas de vegetación, aunque interrumpidas por quebradas donde la concentración 
de nieblas permite el desarrollo de escasas especies de plantas (Luebert \& Pliscoff 2006). Nolana balsamiflua es un arbusto pequeño, endémico de la Región de Antofagasta. Sus flores son hermafroditas, campanuladas, lilas de 2,5-3 cm de longitud, con estambres desiguales de 9-12 $\mathrm{mm}$ y estilos de $5 \mathrm{~cm}$ de longitud (Fig. 1B, C). Florece en diciembre, aunque no existe información precisa de su fenología de floración (Humaña, observación personal). De igual forma, se desconoce el número de flores por individuo. Los frutos están compuestos por 2-3 mericarpos (Eggli 2004).

Estimamos el sistema reproductivo potencial de $N$. balsamiflua mediante cuatro ensayos de polinización manual distribuidos lo más equitativamente posible en 13-26 individuos (Kearns \& Inouye 1993). Si bien se contempló la realización de un control por polinización natural, no fue posible contar con esta información debido a la pérdida de dicho tratamiento experimental. Para estimar si este arbusto es capaz de producir semillas en ausencia de polen (agamospermia), efectuamos una prueba de apomixis en 82 botones florales, los cuales fueron emasculados y embolsados con mallas de tul, hasta la cosecha de semillas, para evitar cualquier contacto entre los estigmas y potenciales polinizadores. Para evaluar si este arbusto es capaz de producir semillas con polen de la misma flor, pero sin mediación de polinizadores (autogamia), efectuamos una prueba de autopolinización automática embolsando 110 botones hasta la cosecha de semillas. Para evaluar la máxima producción de semillas con polen endógeno (geitonogamia), embolsamos 41 botones emasculados, los que fueron polinizados manualmente con polen endógeno ad libitum, de al menos 3 flores, cuando los estigmas estuvieron receptivos. Finalmente, emasculamos 104 botones que luego fueron polinizados manualmente con polen exógeno ad libitum, de al menos 3 individuos creciendo a una distancia $\geq 20 \mathrm{~m}$, para estimar la máxima producción de semillas producidas por exo-cruzamientos (xenogamia).

Transcurridos dos meses, en marzo del año siguiente, registramos la fructificación y el número de semillas producidas por cada flor experimental, pero previo a la dispersión de semillas. Finalmente, estimamos la viabilidad de las semillas mediante la prueba de respiración celular, usando una solución de cloruro de tetrazolio. Para ello, diseccionamos una semilla por fruto producido en cada tratamiento y embebimos el embrión en la solución durante $24 \mathrm{~h}$ en oscuridad. Solo aquellas semillas teñidas fueron consideradas viables (Kearns \& Inouye 1993).
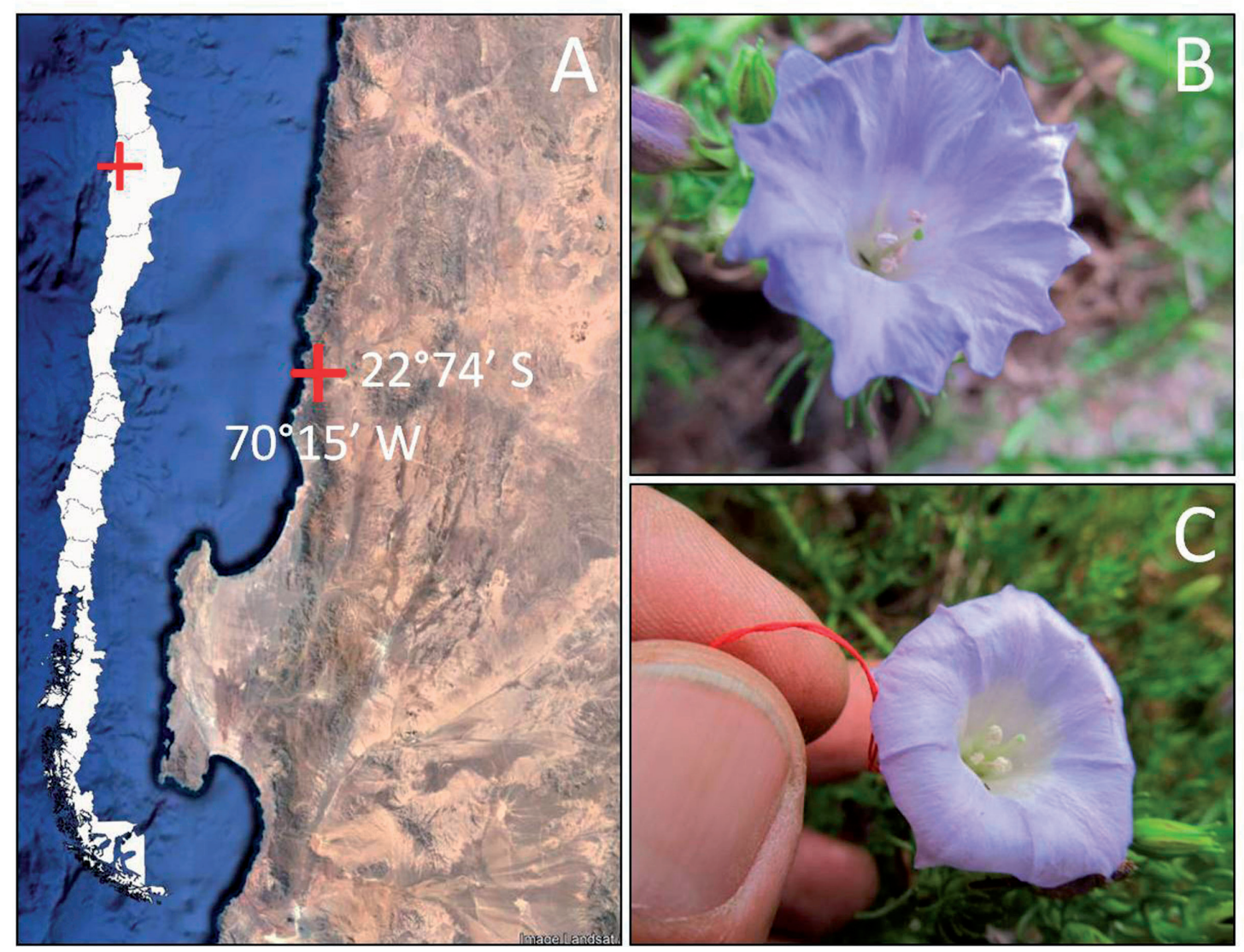

Figura 1. Localización de la "Quebrada del Diablo, Michilla" en la Región de Antofagasta, Chile (A). Allí crece la planta endémica y amenazada del desierto de Atacama Nolana balsamiflua (B, C). Location of the "Quebrada del Diablo, Michilla" in the Antofagasta Region, Chile (A). There the endemic and threatened plant from the Atacama desert, Nolana balsamiflua grows (B, C)" 
La producción de semillas difirió dependiendo de la presencia de polen, el origen de éste, y los vectores polínicos (Tabla 1). De hecho, ninguna flor produjo semillas mediante la prueba de agamospermia, indicando que esta planta requiere de polen para producir semillas (Tabla 1). Las flores tratadas por autogamia y geitonogamia tampoco produjeron semillas, indicando que $N$. balsamiflua es incapaz de reproducirse con polen endógeno, aun si es asistida por polinizadores (Tabla 1). Por el contrario, el ensayo de xenogamia fue el único por el cual la planta fructificó (51,4\% de fructificación), produciendo 1,5 semillas por flor y solo una semilla por mericarpo. El $93,8 \%$ de estas semillas $(n=30)$ fueron viables. Consecuentemente, $N$. balsamiflua es autoincompatible y, por lo tanto, totalmente dependiente de polinizadores para reproducirse.

La nula producción de semillas por autogamia sugiere que esta planta es incapaz de realizar aseguramiento reproductivo, pese a las condiciones ambientales adversas en que habita (Eckert et al. 2006). Esto vuelve a $N$. balsamiflua vulnerable frente a eventuales perturbaciones ambientales, naturales o antropogénicas, restringiendo la posibilidad de mantener poblaciones viables bajo escenarios ecológicos cambiantes (Aizen \& Vázquez 2006, Eckert et al. 2006). Más aun, la profusa floración, con una fructificación máxima cercana al $50 \%$, indica que esta especie realiza un gran esfuerzo reproductivo para atraer polinizadores, produciendo más flores de las que efectivamente pueden fructificar. Desafortunadamente, desconocemos aún la identidad de sus polinizadores y los patrones naturales de fructificación. Estudios adicionales que aumenten el número de plantas estudiadas en distintas locaciones y en distintos años, y que indiquen la diversidad de polinizadores, así como una posible limitación por polen, son fundamentales para respaldar estas conclusiones.

TABLA 1. Número de plantas y flores experimentales, y producción de frutos y semillas en Nolana balsamiflua luego de cuatro ensayos de polinización manual en el desierto de Atacama, Chile. / Number of experimental plants and flowers, and fruit and seed set of Nolana balsamiflua following four hand pollination trials at the Atacama Desert, Chile.

\begin{tabular}{lcccc}
\hline & No. DE Plantas & No. DE FLORES & No. DE FrUtOS/FLOR & No. DE SEMILLAS/FLOR \\
\hline Agamospermia & 21 & 82 & 0 & 0 \\
Autogamia & 26 & 110 & 0 & 0 \\
Geitonogamia & 13 & 41 & 0 & 0 \\
Xenogamia & 23 & 104 & 0,514 & 1,542 \\
\hline
\end{tabular}

Dado el sistema reproductivo y las condiciones de aridez en que crece esta especie, cualquier actividad antropogénica que se ejecute cercana a las poblaciones de $N$. balsamiflua podría afectar fuerte y negativamente su persistencia, no solo por los impactos directos sobre las plantas, sino también por los impactos indirectos vía polinizadores (Aizen \& Vázquez 2006, Eckert et al. 2006). Consecuentemente, la vulnerabilidad que actualmente enfrenta $N$. balsamiflua (Benoit 1989) obliga a la realización de planes de preservación, manejo y restauración, los que deben hacerse considerando sus polinizadores como un elemento clave para el éxito de cualquiera de estas estrategias de conservación.

\section{AGRADECIMIENTOS}

Trabajo financiado por Minera Esperanza mediante el programa de microruteo, rescate y relocalización de flora en Quebrada El Diablo. Agradecemos las facilidades técnicas brindadas por la Universidad de Antofagasta Asistencia Técnica S.A.

\section{REFERENCIAS}

Aizen, M.A., VÁzquEz, D.P. 2006. Flower performance in humanaltered habitats. In: Harder, L.D., Barrett, S.C.H. (eds.), Ecology and evolution of flowers, pp: 159-179. Oxford University Press, New York, USA.

Benoit, I. 1989. Red list of Chilean terrestrial flora. Corporación Nacional Forestal, Santiago de Chile. 151 pp.

Eckert, C.G., SAmis, K.E., DART, S. 2006. Reproductive assurance and the evolution of uniparental reproduction in flowering plants. In: Harder, L.D., Barrett, S.C.H (eds.), Ecology and evolution of flowers, pp. 183-203. Oxford University Press, New York, USA.

EgGLI, U. 2004. Illustrated Handbook of Succulent Plants: Dicotyledons. Springer, pp. 326-332.

GonzÁlez, A.V., Pérez, F. 2010. Pollen limitation and reproductive assurance in the flora of the coastal Atacama Desert. International Journal of Plant Science 171: 607-614.

Kearns, C.A., Inouye, D.W. 1993. Techniques for pollination biologists. University Press of Colorado, Colorado. i-xviii $+583 \mathrm{pp}$.

Luebert, F., Pliscoff, P. 2006. Sinopsis bioclimática y vegetacional de Chile. Editorial Universitaria, Santiago de Chile. 316 pp.

Richards, A.J. 1997. Plant breeding system. Chapman and Hall, London. xii $+529 \mathrm{pp}$.

Recibido: 02.04.2018

Aceptado: 07.11.2018 